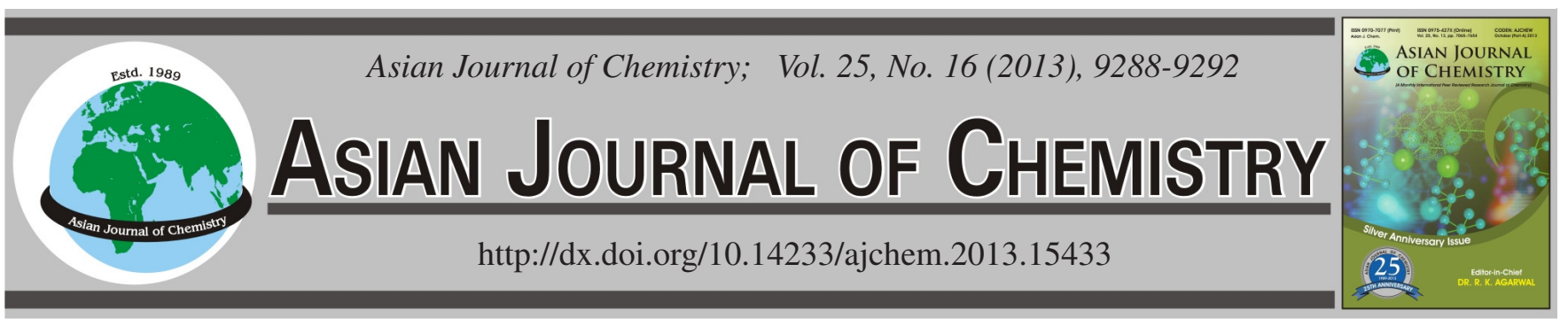

\title{
Study of Kinetics on Liquid-Phase Catalytic Oxidation of Toluene
}

\author{
G. PENG ${ }^{1, *}$, E.V. DAVIS ${ }^{2}$ and F. WEN ${ }^{3}$
}

${ }^{1}$ School of Chemical Engineering, Ningbo University of Technology, Ningbo 315016, P.R. China

${ }^{2}$ Department of Chemistry, Clemson University, Clemson 29631, USA

${ }^{3}$ Department of Chemical Engineering, Zhejiang University, Hangzhou 310007, P.R. China

*Corresponding author: E-mail: gepengv@ hotmail.com

(Received: 6 March 2013;

Accepted: 20 September 2013)

AJC-14163

\begin{abstract}
The effects of initial concentration,catalyst dosage and catalyst proportion on the result of oxidation of toluene were investigated and kinetic models were figured out. It could be considered that the oxidation rate of toluene wasn't varied significantly with the change of toluene concentration. The highest yield of benzaldehyde increased with the decrease of toluene concentration and the increase of catalyst dosage. The catalyst proportion had no significant influence on the yield of benzaldehyde and the oxidation of toluene could be inhibited by increasing $\mathrm{Co} / \mathrm{Mn}$. There was no significant difference between the activation energies of toluene and benzaldehyde.
\end{abstract}

Key Words: Kinetics, Liquid-phase oxidation, Toluene, Benzaldehyde.

\section{INTRODUCTION}

The liquid-phase oxidation of toluene can be retrieved to the 1960's and Rhone-Poulenc Company in France ${ }^{1}$ obtained phenyl methanol, benzaldehyde and benzene carboxylic acid by liquid-phase air oxidation of toluene. Many scholars ${ }^{2-7}$ argue now that the homogeneous catalytic oxidation of toluene abides by the mechanism of free radical reaction. Firstly, toluene is transformed into peroxide and then decomposed into free radicals under the functions of the catalyst, in the meantime, metal ions can realize the oxido-reduction cycle and function in transporting electrons and accomplish electron transfer ${ }^{8,9}$. The present study carried out investigations on the kinetics of liquid-phase catalytic oxidation of toluene and the mechanisms of its oxidation reaction.

\section{EXPERIMENTAL}

The experiment was carried out in different batches, firstly the reaction materials, solvents, catalysts were prepared into a solution of $300 \mathrm{~mL}$ in proper proportions. The solution was added into the reactor, the air in the reactor was exhausted by introducing nitrogen gas after tightly sealing. Pressurization was then carried out with nitrogen gas to a final pressure at about $1 \mathrm{MPa}$, the inlet valve and the outlet valve were shut off, the power supply was switched on for heating, subsequently the valve for cooling water in the end gas condensation tube at the same time and the stirring was started. Nitrogen gas was continuously introduced during the process for increasing temperature in order to guarantee the synchronized increase in pressure and temperature. After the temperature and the pressure increased to the preset values, the heating power was adjusted in order to maintain the temperature in the reactor constant and the stirring speed was adjusted to $900-950 \mathrm{rpm}$. Finally the nitrogen gas was switched to the air and the reaction was triggered.

Analytical method: Sampling was carried out for every short period during the reaction and the ingredients in the sample were determined by using a HP6890-MS5973 gas chrom-atograph-mass spectrometer and quantitative analysis was carried out by using a Shimazu GC-9A gas chromatograph. The chromatographic column was a SE-54 capillary column and the detector was a hydrogen flame ion detector (FID).

\section{RESULTS AND DISCUSSION}

The differential equation for the changes in the concentrations of different ingredients during the oxidation of toluene with the time lapsed was as below:

$$
\frac{\mathrm{dc}_{1}}{\mathrm{dt}}=-\mathrm{r}_{1} \frac{\mathrm{dc}_{2}}{\mathrm{dt}}=\mathrm{r}_{1}-\mathrm{r}_{2} \frac{\mathrm{dc}_{3}}{\mathrm{dt}}=\mathrm{r}_{2}
$$

The model formula (1) for the kinetics of hyperbolic curve during the liquid-phase oxidation of aromatic methyl hydrocarbon was proposed by analyzing the reaction mechanism for methyl aromatic hydrocarbon in depth, but there were too many parameters in the formula (1) and some simplifications were required. During the liquid-phase catalytic oxidation of 
toluene, the concentrations of free radicals and trivalent metal catalysts were very low and the changes were slow, which met the hypothesis of pseudo-steady state. Therefore, [M(III)], $[\mathrm{I} \cdot],[\mathrm{M}(\mathrm{II})]$ and $\left[\mathrm{Br}^{-}\right]$can be all considered as constants. Furthermore, many concentrations of higher order term can be found in the formula (1) and these concentrations were all very low, thus these high order terms can be omitted. Therefore, the simplified model for the mechanism by these simplifications was as followed:

$$
\begin{aligned}
& \mathrm{r}_{1}=\frac{\mathrm{k}_{1}\left[\mathrm{RCH}_{3}\right]}{\mathrm{d}_{1}\left[\mathrm{RCH}_{3}\right]+\mathrm{d}_{2}[\mathrm{RCHO}]+1} \\
& \mathrm{r}_{2}=\frac{\mathrm{k}_{2}[\mathrm{RCHO}]}{\mathrm{d}_{1}\left[\mathrm{RCH}_{3}\right]+\mathrm{d}_{2}[\mathrm{RCHO}]+1}
\end{aligned}
$$

Effects of the original concentration on toluene oxidation: Benzene carboxylic acid was used as the solvent and a series of experiments were carried out by selecting different incipient concentrations of toluene [toluene: benzene carboxylic acid $=2: 1,1: 1,1: 3,1: 5$ and 1:10 (mass:mass) $]$ and other conditions were not changed $([\mathrm{Co}]=300 \mathrm{ppm},[\mathrm{Co}]:[\mathrm{Mn}]:[\mathrm{Br}]=$ 1:1:1 (mol:mol:mol), $\mathrm{P}=1.7 \mathrm{MPa}, \mathrm{T}=170{ }^{\circ} \mathrm{C}$ ). The results were shown in Fig. 1, the concentrations in the figure were all normalized concentrations, namely, the concentrations were obtained by dividing the concentrations with corresponding incipient concentrations of toluene. The results were shown in Figs. 1-3.

It can be found in Fig. 2 that the time for toluene to achieve the same transformation rate gradually decreased with the decrease in the incipient concentration of toluene. However, if the rules of first-order reaction were met, the changing curves of normalized concentrations of toluene at different original concentrations should be overlapped since the changes of concentrations cannot change the rate constant $\mathrm{k}_{\mathrm{i}}$ value and the apparent order for toluene from the results in Fig. 2 approached the zero-order reactions. With the decrease in the incipient concentration of toluene, the highest yield of benzaldehyde continuously increased and the selectivity of benzaldehyde also showed an increasing tendency and the reaction time for benzaldehyde to reach the highest yield was shortened. When

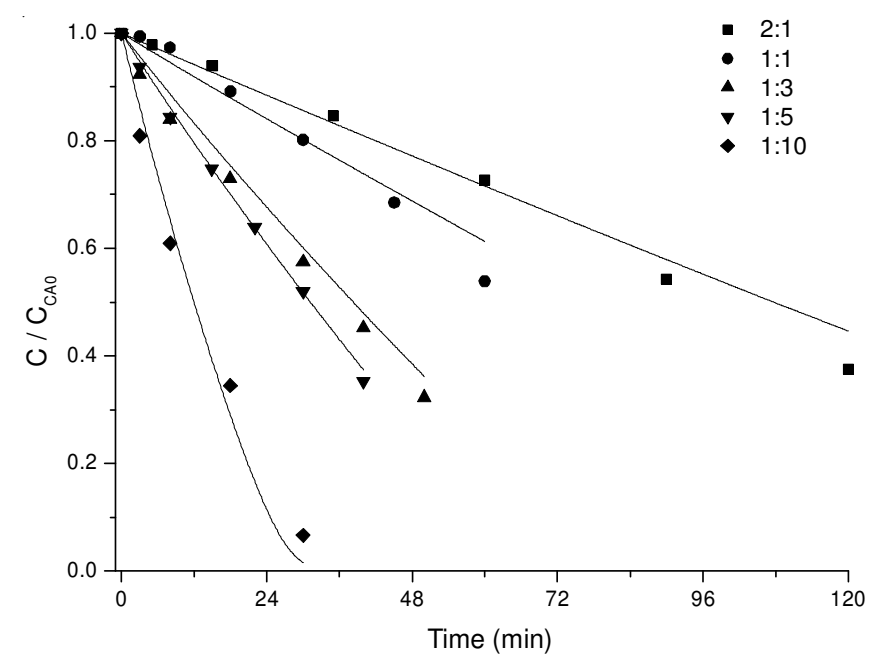

Fig. 1. Toluene concentration changes with different initial concentration and time

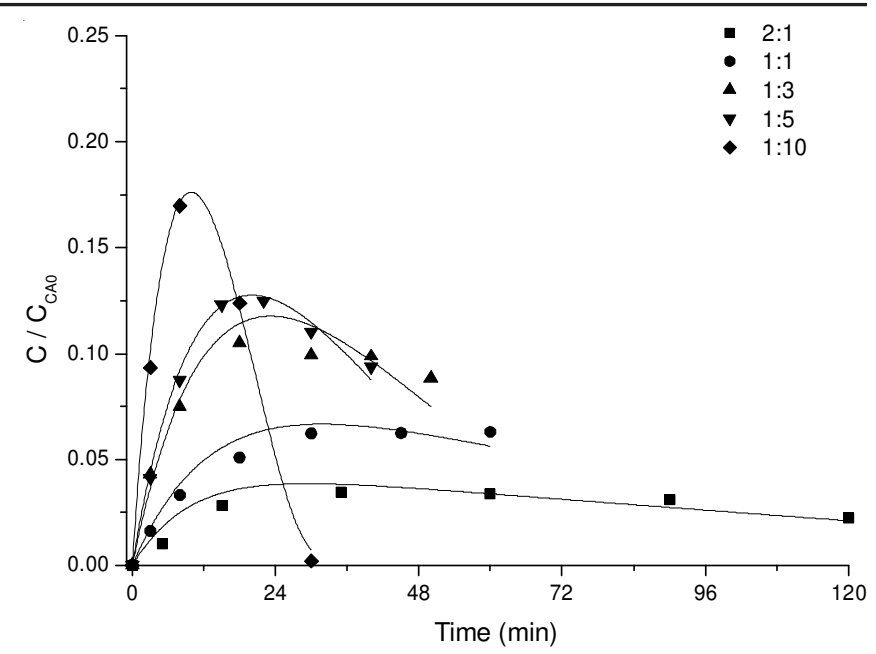

Fig. 2. Benzaldehyde concentration changes with different initial concentrations and time

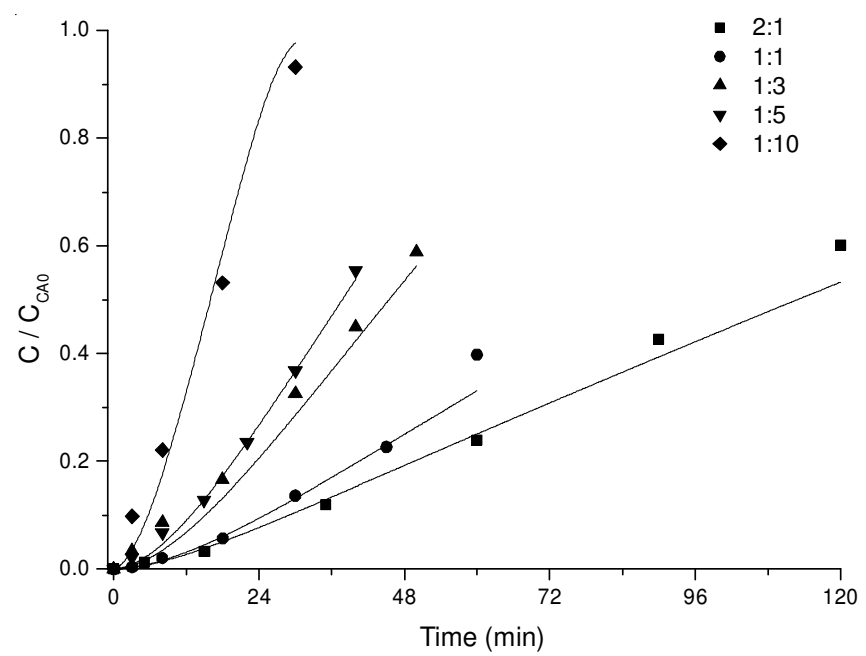

Fig. 3. Benzoic acid concentration changes with different initial concentrations and time

the ratio of toluene:benzene carboxylic acid $=1: 10$, the time of benzaldehyde to reach the highest yield was $8 \mathrm{~min}$ and the highest yield was $17 \%$. The selectivity of benzaldehyde at this time was $43.5 \%$. Therefore, the operations should be carried out at a low temperature in order to obtain benzaldehyde in a relatively high yield.

Effects of catalyst concentration on toluene oxidation: The incipient concentration of toluene, temperature and pressure for the reaction as well as the proportion of catalysts were fixed and the experiment was carried out by selecting different catalyst concentrations. The experimental results were subjected to fitting by using the kinetic model proposed previously in the present study and the rate constants $k_{i}$ in different steps at different catalyst concentrations were obtained and the results for liquid-phase catalytic oxidation of toluene and the model fitting were shown in Figs. 4-6.

With the increase in catalyst concentration, the reaction rate increased and the highest yield of benzaldehyde also gradually increased, the time for benzaldehyde to reach the highest yield was gradually cut down. Furthermore, the selectivity of benzaldehyde at the highest yield also was increased. When the concentration of cobalt was 900 ppm, the highest 


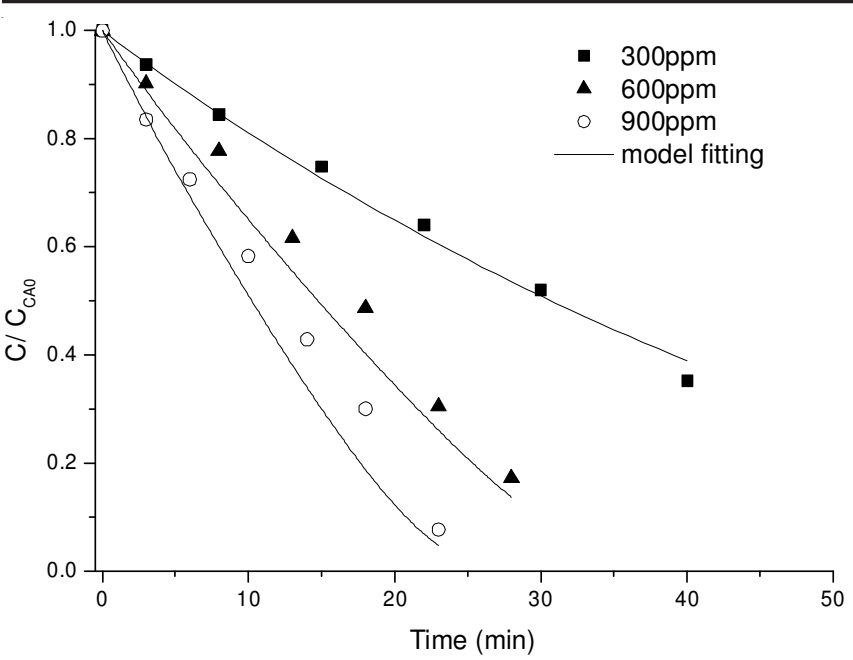

Fig. 4. Toluene concentration changes with different catalyst concentrations and time

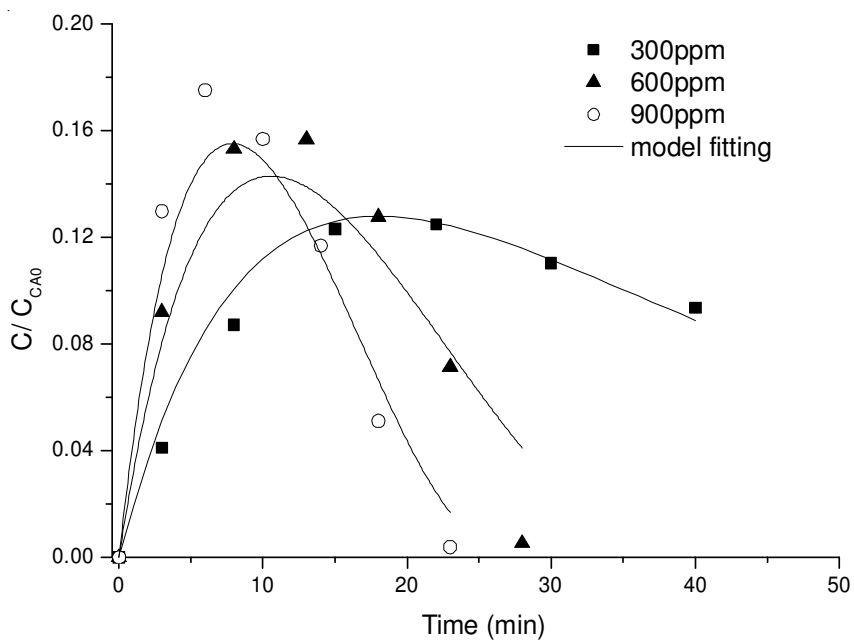

Fig. 5. Benzaldehyde concentration changes with different catalyst concentrations and time

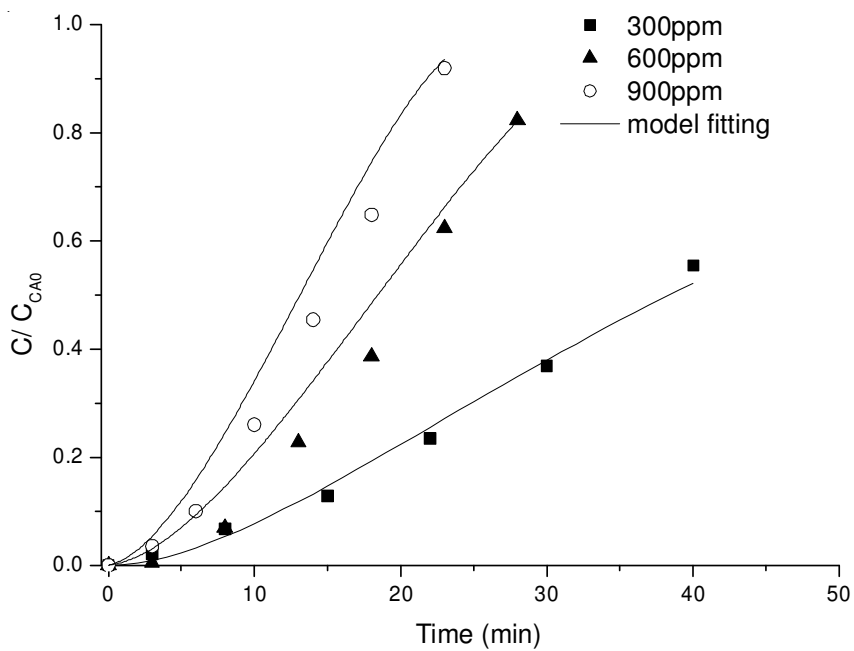

Fig. 6. Benzoic acid concentration changes with different catalyst concentrations and time

yield of benzaldehyde was about $18 \%$ and the selectivity of benzaldehyde was $64 \%$ and the time for benzaldehyde to reach the highest yield was $6 \mathrm{~min}$. The functions of catalyst concentration in improving the yield of benzaldehyde was significant, normally the increase in the total concentration of cobalt, manganese and bromine can increase the rate of aromatic hydrocarbon oxidation, while toluene is a typical chain reaction, the increase in the total concentration of catalysts was beneficial for improving the rate of toluene oxidation and promoting benzaldehyde oxidation, but the effects of total concentration of catalysts on the rate of toluene oxidation were much significant than the effects on aldehyde group oxidation, which also led to the increase in the highest yield of benzaldehyde.

The curves of the changes on the reaction-rate constants at different steps with the catalyst concentrations were shown in Fig. 7. It can be found from the figure that the reaction rate constants at different steps increased with the increase in catalyst concentrations and the sensitivity for $\mathrm{k}_{2}$ to the change in catalyst concentrations within the range of high catalyst concentrations. But the sensitivity for $\mathrm{k}_{1}$ to the change in catalyst concentrations almost did not change, $\mathrm{k}_{1}$ increased by three times when the catalyst concentration increased by two times, but $\mathrm{k}_{2}$ only increased by 2.5 times, that is to say, the effects for the increase in catalyst concentration on the improvement in the rate of toluene oxidation were significant than those on toluene oxidation. Thus it also led to the increase in the intermediate product benzaldehyde with the increase in catalyst concentration. Therefore, the operations should be carried out at high catalyst concentrations in order to obtain benzaldehyde in a relatively high yield.

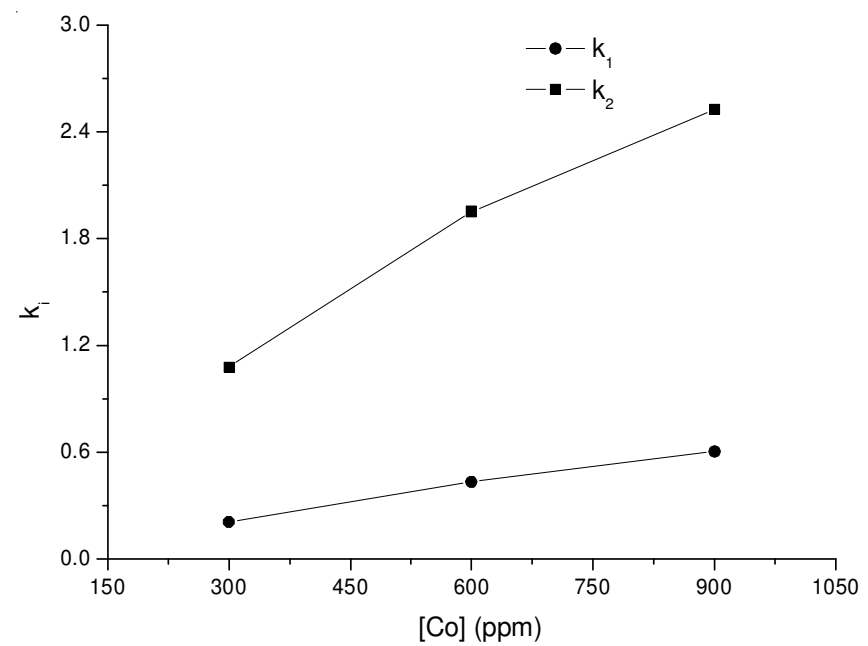

Fig. 7. Each step reaction rate constant changes with the catalyst concentrations

Effects of catalyst proportion on Toluene oxidation: Under the effects from catalytic composites, the effects of cobalt and manganese on aromatic hydrocarbon oxidation were normally different, thus the effects of different proportions between cobalt and manganese on liquid-phase catalytic oxidation of toluene were examined here. The incipient concentration of toluene [toluene:benzene carboxylic acid= 1:5 (mass: mass)], reaction temperature $\left(170{ }^{\circ} \mathrm{C}\right)$, pressure $(1.7 \mathrm{MPa})$, $[\mathrm{Br}]=408 \mathrm{ppm}$ and $[\mathrm{Co}+\mathrm{Mn}]:[\mathrm{Br}]=2: 1(\mathrm{~mol}: \mathrm{mol})$ were fixed and the proportions in catalysts were changed ([Co]:[Mn] $=1: 1,3: 1,8: 1(\mathrm{~mol}: \mathrm{mol}))$. The results were shown in Figs. 8-10. 


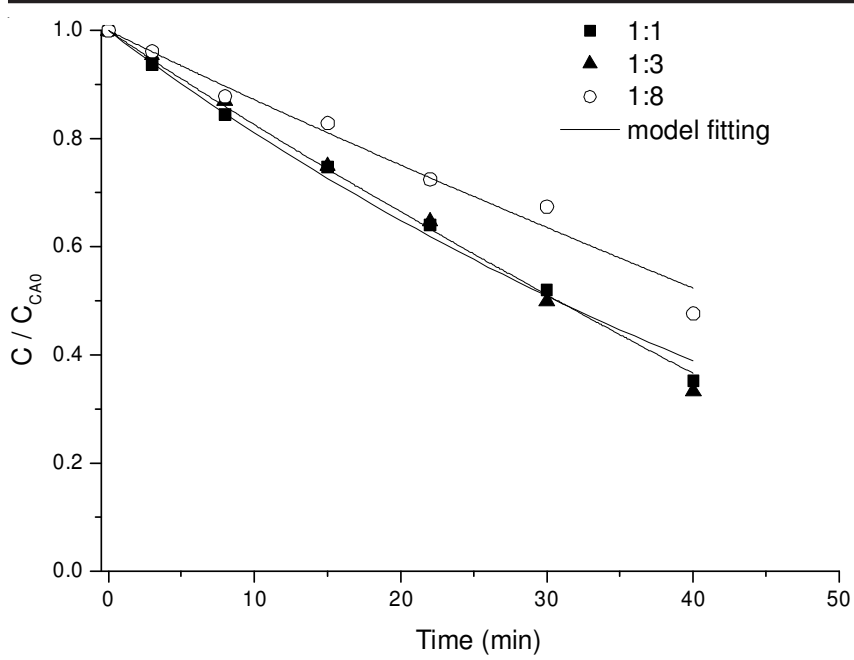

Fig. 8. Toluene concentration changes with different catalysts and time

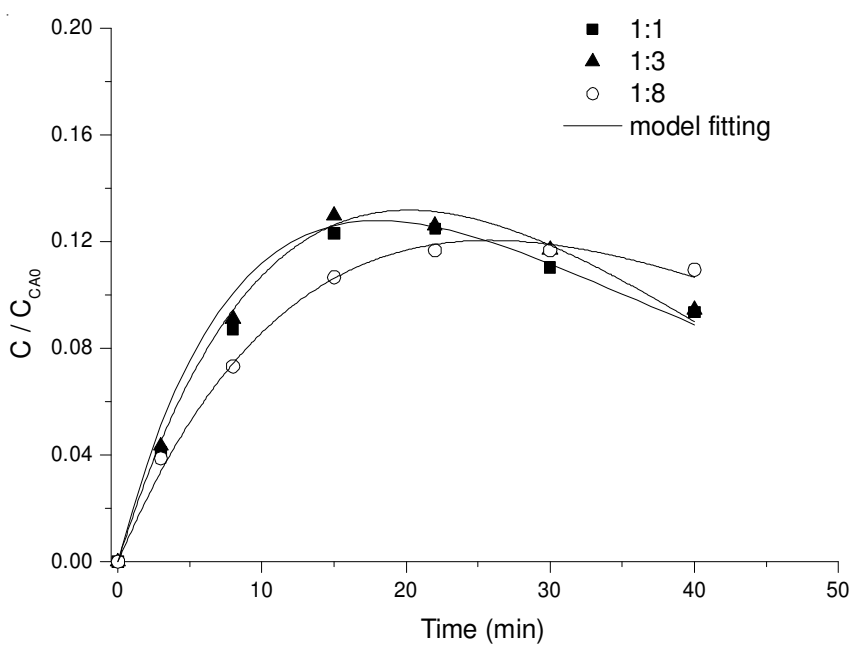

Fig. 9. Benzaldehyde concentration changes with different catalysts and time

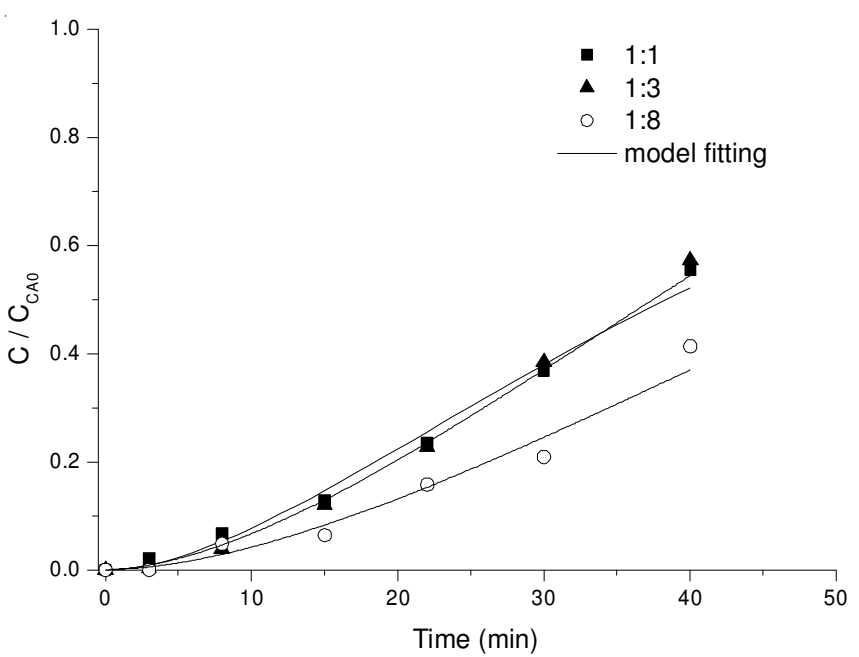

Fig. 10. Benzoic acid concentration changes with different catalysts and time

Figs. 8-10 show that the change in the proportion of catalysts had no significant effects on toluene oxidation when the proportion of catalyst [Co]:[Mn] $=1: 1$ and 3:1. However, the convert rate of toluene oxidation significantly decreased when the concentration of cobalt further increased to $[\mathrm{Co}]:[\mathrm{Mn}]=$
8:1 and the highest yield of benzaldehyde also decreased accordingly. Previous investigations have shown that cobalt mainly promoted methyl oxidation during the process of methyl oxidation, while manganese mainly promoted the oxidation of aldehyde. However, it can be found from the results that sole decrease in manganese concentration can also decrease the rate of methyl oxidation and the decrease in methyl oxidation rate was faster than that of aldehyde oxidation. Therefore, sole decrease in manganese concentration was not advantageous for improving the yield of benzaldehyde and the synergistic actions of cobalt and manganese were much significant than the effects of merely cobalt or manganese on toluene.

The curves of the changes in the reaction-rate constants at different steps with the changes in catalyst proportion were shown in Fig. 11. It can be easily found that the reaction rate constant of toluene began to slightly increase with the gradual increase in the ratio between cobalt and manganese, but $\mathrm{k}_{1}$ began to decrease significantly when the ratio between cobalt and manganese continuously increased, while the reactionrate constant of benzaldehyde almost did not change at the beginning with the increasing of the ratio between cobalt and manganese. It may seriously affect the oxidation of toluene when the ratio between cobalt and manganese was higher than 3 and the reaction rates of toluene at different steps all significantly decreased and the highest yield of benzaldehyde also showed certain decreased.

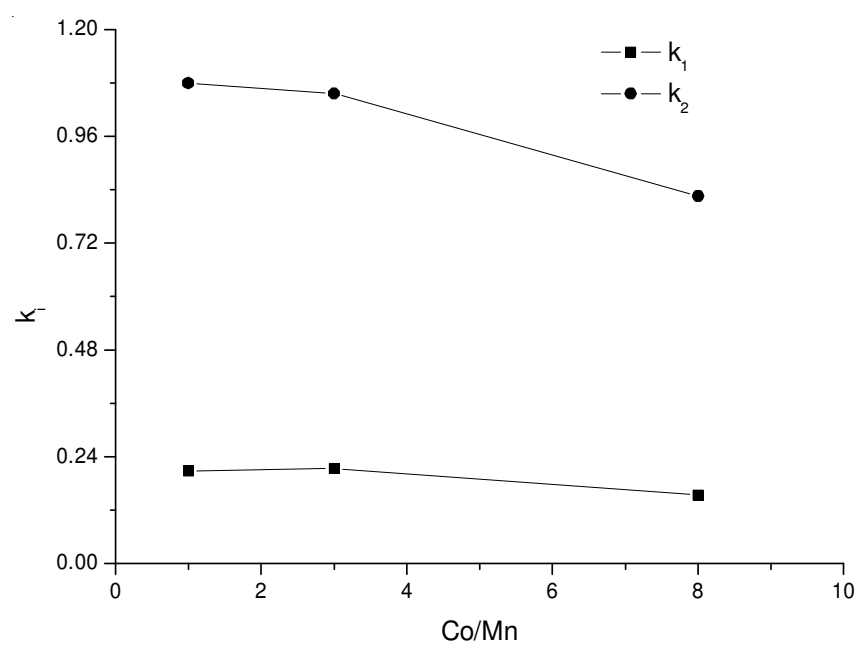

Fig. 11. Reaction rate constant $\mathrm{k}_{2}$ linearity in different temperatures

\section{Conclusion}

The change of the incipient concentration of toluenehad slightly effects on the rate of toluene oxidation and it followed the kinetic rules with an apparent order with zero grade. The highest yield of benzaldehyde increased with the decreasing in the original concentration of toluene. The increasing of catalyst concentration can increase the reaction rates of toluene oxidation at different reaction steps and the sensitivity of the rate of toluene oxidation with the change of catalyst concentration was higher than that of the rate of benzaldehyde oxidation. The operations should be carried out at high catalyst concentrations in order to obtain relatively high yield of benzaldehyde. The changes of catalyst proportion can affect toluene oxidation to some extent, but the increasing of the ratio between cobalt 
and manganese can slightly increase the highest yield of benzaldehyde when Co:Mn was lower than 3, but continuous increasing of the ratio between cobalt and manganese can decrease the reaction rates of toluene oxidation at different reaction steps when Co:Mn was higher than 3 and it was not disadvantageous to improve the yield of benzaldehyde.

\section{Nomenclature}

$$
\begin{aligned}
& \mathrm{t}=\text { Time }(\min ) \\
& \mathrm{C}=\text { Mole concentration }(\mathrm{mol} / \mathrm{L}) \\
& \mathrm{k}_{\mathrm{i}}=\text { The rate contants } \\
& \mathrm{r}=\text { Reaction rate }(\mathrm{mol} / \mathrm{min} \mathrm{L})
\end{aligned}
$$

\section{REFERENCES}

1. R.D. Zhao, Aromatic Hydrocarbon Technology, Chemical Industry Press, Beijing (2001).

2. G. Peng, Asian J. Chem., 25, 735 (2013).

3. S.S. Kamath and S.B. Chandalia, J. Appl. Chem. Biotechnol., 23, 469 (1973).

4. M.L. Kantam, P. Sreekanth, K.K. Rao, T.P. Kumar, B.P.C. Rao and B.M. Choudary, Catal. Lett., 81, 223 (2002).

5. K. Auty, B.C. Gilbert, C.B. Thomas, S.W. Brown, C.W. Jones and W.R. Sanderson, J. Mol. Catal., 117, 279 (1997).

6. H.V. Borgaonkar and S.B. Chandalia, J. Chem. Technol. Biotechnol., 34, 107 (1984).

7. H.V. Borgaonkar, S.R. Raverkar and S.B. Chandalla, Ind. Eng. Chem. Prod. Res. Dev., 23, 455 (1984).

8. X.D. Jiao, P.D. Metelski and J. Espenson, Inorg. Chem., 40, 3228 (2001).

9. E.J.Y. Scott and A.W. Chester, J. Phys. Chem., 76, 1520 (1972). 